der anstrengungsinduzierten Weizenallergie ein wichtiges diagnostisches Instrument, betonte Grosber.

\section{Vier Stunden Pause nach dem Essen}

In der Patientenberatung ist auf einen mindestens vierstündigen Abstand zwischen dem Verzehr weizenmehlhaltiger Lebensmittel und körperlicher Anstren- gung hinzuweisen. Zudem sollten die Patienten individuelle weitere Auslöser meiden sowie mögliche Kreuzreaktionen beachten. Bei einer unübersichtlichen Situation bezüglich möglicher Kofaktoren sollte eine Weizenkarenz empfohlen werden, so Grosber. Zudem sind die Patienten mit einem Notfallset auszustatten.
Fischer J, Biedermann T: Mit Omega-5-Gliadin assoziierte Anstrengungs-induzierte Weizenallergie bei einer Europäerin. Grosber M, Ollert M, Engst R, Simonowa A, Huss-Marp J, Schmitt $M$, McIntyre $M$, Ring J, Darsow U: Anstrengungsinduzierte Weizenanaphylaxie und Omega-5Gliadin. 20. Mainzer Allergie-Workshop, Mainz, 7./8. März 2008

\title{
Wie Stallstaubbakterien vor Asthma schützen
}

\section{Eine Exposition der werdenden Mutter gegenüber Mikroben aus Stallstaub scheint den Nachwuchs vor Asthma zu bewahren. Zumindest im Mausmodell kommt es dabei zu protektiven epigenetischen Modifikationen.}

E pidemiologische Daten zeigen, dass Kinder, die auf traditionellen Bauernhöfen aufwachsen, deutlich seltener als andere Kinder unter respiratorischen Allergien leiden. Möglicherweise hat bereits die pränatale Exposition gegenüber verschiedenen mikrobiellen Komponenten, denen die werdende Mutter durch den Kontakt zu den Tieren ausgesetzt ist, einen Einfluss auf die Allergieentwicklung beim Kind. Eine Marburger Arbeitsgruppe konnte in den Stäuben von Tierställen zwei Bakterienstämme identifizieren, die dieses Milieu vorrangig besiedeln: das gramnegative Proteobakterium Acinetobacter lwoffii und das grampositive Milchsäurebakterium Lactococcus lactis. Im Mausmodell des experimentellen Asthmas hatten sie allergieprotektive Eigenschaften, berichtete Dr. René Teich. Nun wurde überprüft, ob ein Transfer der Allergieprotektion von der Mutter auf die Nachkommen stattfindet und die pränatale Exposition gegenüber $A$. lwoffii eine Asthmaentwicklung bei den Nachkommen verhindert.

Nachkommen $A$. lwoffii-exponierter Mäuse wiesen im Vergleich zu Nachkommen von Kontrollmäusen nach Sensibilisierung mit Ovalbumin eine normalisierte Lungenfunktion und signifikant geringere Atemwegsentzündungen auf. Dieser

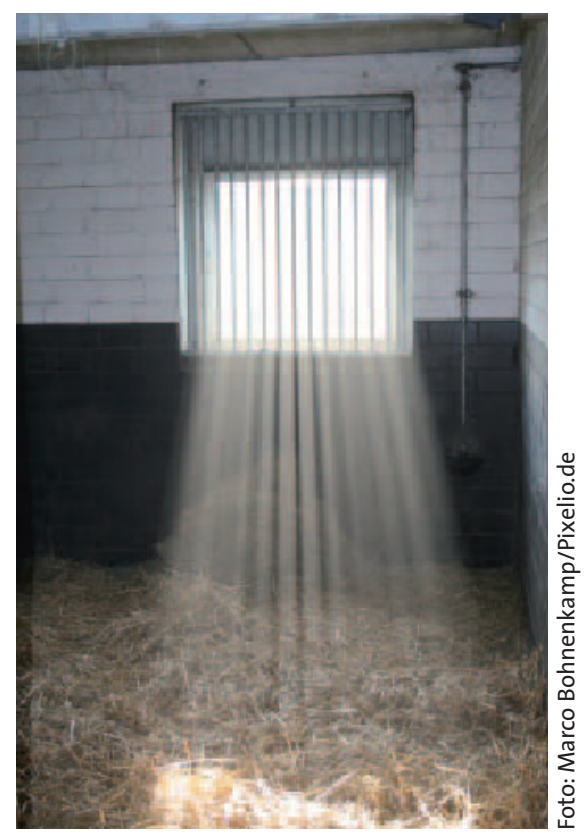

Noch ist die genaue Rolle des Stallstaubs nebulös.

Befund war mit einer hochregulierten Produktion proinflammatorischer Zytokine und einem reduzierten TH2-Zytokinlevel bei den Nachkommen assoziiert. Somit konnte gezeigt werden, dass die pränatale Exposition gegenüber den Stallbakterien bei den Nachkommen die angeborene wie auch adaptive Immunant- wort moduliert und präventiv gegenüber Allergien der Atemwege wirkt.

\section{Es liegt auch auf den Genen}

Einen möglichen Mechanismus stellte Stephanie Brand aus derselben Arbeitsgruppe vor. Man weiß, dass epigenetische Modifikationen im Rahmen von GenUmwelt-Interaktionen vorkommen, die die Genexpression beeinflussen können. Die Forscher gingen jetzt der Frage nach, ob die A.-lwoffii-Exposition mit einer veränderten DNA-Methylierung im IFN- $\gamma$-Genlocus von T-Zellen einhergeht. Bei Mäusen, die vor und während der Trächtigkeit intranasal mit $A$. lwoffii behandelt wurden, konnte mittels Pyrosequenzierung eine Erniedrigung der Methylierung im IFN- $\gamma$-Promotor gezeigt werden, die eine erhöhte IFN- $\gamma$ Produktion durch in vitro restimulierte mononukleäre Zellen aus der Milz zur Folge hatte. Bei den Nachkommen dagegen kam es zu einer Erhöhung des Methylierungsgrades im IFN- $\gamma$-Promotor von T-Zellen, die mit einer verminderten IFN- $\gamma$-Produktion nach Restimulation einherging. Somit konnte ein epigenetischer Mechanismus aufgezeigt werden, der an der Asthmaprotektion beteiligt ist, schlussfolgerte Brand: Infolge der $A$.-lwoffii-Exposition komme es zu einer Regulierung der IFN- $\gamma$-Produktion durch Änderung der DNA-Methylierung in dessen Promotorbereich. abd

Teich R, Blümer N, Patrascan C, Garn H, Renz H. Allergie-Prävention mittels mikrobieller Komponenten aus Staubextrakten.

Brand S, Teich R, Garn H, Renz H: Methylierung des IFN-gamma-Promotors als möglicher Mechanismus der Allergieprotektion durch Acinetobacter Iwoffii. 20. Mainzer Allergie-Workshop, Mainz, 7./8. März 2008 